Mund Kiefer GesichtsChır (1997) 1:189 C Sprınger-Verlag 1997

\section{Prof. Dr. Dr. Rüdiger Becker zum 70. Geburtstag}

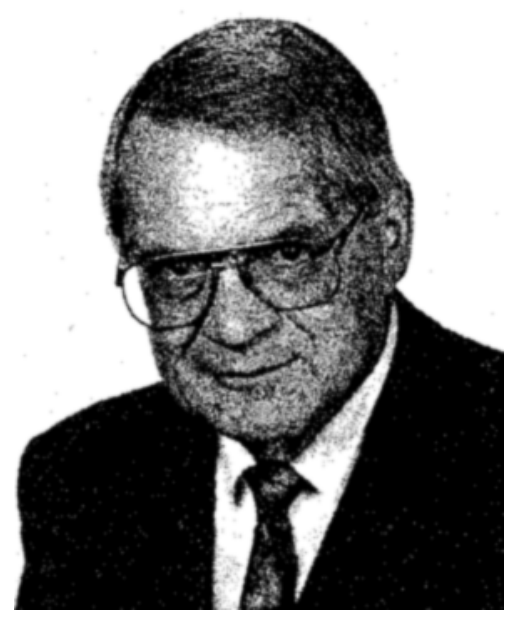

Prof. Dr. Dr. R. Becker, emeritierter Direktor der Klinik und Poliklinik für Mund-, Kiefer- und Gesichtschirurgie in Münster, vollendete am 12.4. 1997 sein 70. Lebensjahr.

Er war seit 1971 Lehrstuhlinhaber für Mund-, Kiefer- und Gesichtschirurgie und ab 1972 Direktor des Zentrums für Zahn-. Mund- und Kieferheilkunde der Westfälischen WilhelmsUniversität Münster.

Prof. Dr. U. Joos

Univ-Zahnklink,

Direktor der Klınık für MKG-Chırurgıe,

Waldeyerstraße 15, D-48149 Münster
Unter seiner Federführung wurde die neue Münsteraner Zahnklinik, eine der größten Europas, geplant und in Betrieb genommen. Während seiner Amtsperiode konnte er 8 Habilitationen erfolgreich beenden, mehrere Lehrstühle und Chefarztpositionen besetzen und zahlreiche Dissertationen abschließen.

Im universitären Bereich engagierte sich Herr Prof. Becker als Prodekan und Dekan der Medizinischen Fakultät sowie als Mitglied des Senats der Westfälischen Wilhelms-Universität Münster.

Wissenschaftlich befaßte er sich hauptsachlich mit der Behandlung von Störungen des Kieferwachstums und der Rekonstruktion von Kiefer- und Gesichtsdefekten. Weit über $100 \mathrm{Pu}$ blikationen zu diesen Gebieten stammen aus seiner Feder, dazu zahlreiche Buchbeiträge und eine Vielzahl von Vorträgen. Seine herausragende wissenschaftliche Tätigkeit fand ihre Würdigung in der Ehrenmitgliedschaft des renommierten Royal College of Dental Surgeons und der Deutschen Gesellschaft für Mund-, Kiefer- und Gesichtschirurgie, der Aufnahme in die Deutsche Akademie der Naturforscher ,Leopoldina" sowie der Berufung als Gutachter in die Sachverständigenkommission der Deutschen Forschungsgemeinschaft. Als langjähriger Präsident der Deutschen Gesellschaft für Mund-. Kiefer- und Gesichtschirurgie hat er entscheidend zur Weiterentwicklung dieser Fachdisziplin beigetragen.

U. Joos, Münster
Die Redaktion der ,Mund-, Kieferund Gesichtschirurgie" möchte ausdrücklich dem früheren Chefredakteur der Deutschen Zeitschrift für Mund-, Kiefer- und Gesichtschirurgie zum Geburtstag recht herzlich gratulieren. Die fast zwei Dekaden währende hochqualifizierte Redaktionsarbeit von Herrn Prof. Becker ist die Basis dafür gewesen, daß wir ein anerkanntes Journal heutzutage führen dürfen. Auf ausdruicklichen Wunsch des Jubilars verzichtet der Unterzeichner auf eine Laudatio, wie sie Herm Prof. Becker zum 60. Geburtstag und zum 65. Geburtstag sowie zur Ernennung seiner Ehrenmitgliedschaft innerhalb der Deutschen Gesellschaft für Mund-, Kiefer- und Gesichtschirurgie im vergangenen Jahr gewidmet wurde und wie sie aus Sicht der Redaktionsarbeit angemessen ist. Die Gratulation seiner Freunde und Schüler sollte aber dennoch in dieser Sequenz mit den besten Wünschen für eine gesunde Zukunft an dieser Stelle formuliert werden.

Für die Redaktion E. Machtens, Bochum 